\title{
Lithospheric wide-angle seismic profiles using stacked airgun shots
}

\author{
Deping Chian \\ Geological Survey of Canada (Atlantic), Dartmouth, Nova Scotia, Canada
}

\section{Jeremy Hall}

Department of Earth Sciences, Memorial University of Newfoundland, St. John's, Newfoundland, Canada

\author{
François Marillier \\ Geological Survey of Canada (Atlantic), Dartmouth, Nova Scotia. Canada
}

\begin{abstract}
The signal-to-noise ratio of marine wide-angle seismic profiles can be significantly enhanced by stacking multiple shots. Signals detected from airgun shots from a seismic ship repeated many times within a small source area (called "stack shots") can be stacked in a manner somewhat similar to common-mid-point processing of reflection seismic data. We collected two such "stack shot" profiles across the eastern margin of Newfoundland. At each shooting site, 36 closely spaced airgun shots were fired consecutively, and recorded along a profile made of about 400 land receivers at offsets of 100 to $455 \mathrm{~km}$. While the data can be stacked in several different ways we show that a two-step technique or "two-pass stack" is the most effective. The traces of each receiver gather are first stacked at an aperture of $0.5 \mathrm{~km}$ along a slope of $8 \mathrm{~km} / \mathrm{s}$ (stacking with linear moveout). The stacked traces are then reordered by increasing offset and stacked a second time along the same slope and with an aperture of 0.5 $\mathrm{km}$ or less. This technique is superior to a direct stack in which all the traces would be stacked in one pass because it allows improvement in the data quality by the various methods designed for each phase of the two-pass stack. Our results show that the "stack shot" technique coupled with the "two-pass stack" is a viable alternative, with less environmental impact, to using large, single explosions at sea.
\end{abstract}

\section{Introduction}

Controlled-source seismic experiments for studying the Earth's interior can be divided into two categories, i.e. one focused on near-vertical reflections and one on wide-angle reflections/refractions. Near-vertical reflections are routinely used in the oil industry, and the method is characterized by extensive overlap of seismic raypaths giving a high level of data redundancy, which allows significant enhancement of signals by "stacking" and imaging of the subsurface through migration. On the other hand, the wide-angle method has been used routinely to study the velocity structure at greater lithospheric depths. But this method is usually associated with much lower redundancy due to very low, or a lack of, overlap of seismic rays. As a consequence, enhancement of signal-to-noise $(\mathrm{S} / \mathrm{N})$

\section{Copyright 1996 by the American Geophysical Union.}

Paper number 96GL02070

0094-8534/96/96GL-02070\$05.00 ratios is achieved only by stacking of a few adjacent traces (e.g. Chian and Louden. 1992), or by increasing the size of energy sources and thus of potential damage to the environment.

This paper presents a special design of a wide-angle seismic experiment that we call the "stack shot" technique in which the number of adjacent traces for stacking is greatly increased. While simple stacking can enhance the seismic signals (e.g. Neprochnov et al., 1985), we show that a new, two-pass stacking algorithm produces higher accuracy and quality.

\section{Data}

One of the objectives of the seismic refraction/wide-angle reflection experiment conducted in 1991 as part of the Lithoprobe East project across the Newfoundland Appalachian orogen was to record wide-angle seismic arrivals from the upper mantle. "Stack shot" profiles were collected to complement other crustal profiles gathered in this area (Marillier et al., 1994; Hughes et al., 1994).

About 400 receivers were deployed at approximately 500 metre intervals on land to record seismic signals from two series of "stack shots" at sea. The receivers were digital instruments from the Geological Survey of Canada (Scintrex EDA PRSs) and from the US Geological Survey. For each series of shots, an array of sIx airguns towed by a ship moving at 2 knots along the line were fired every minute for 36 minutes. The array had a total volume of $98.4 \mathrm{~L}\left(6000 \mathrm{in}^{3}\right)$ and was operated at $13.8 \mathrm{MPa}$ (2000 psi ). The shots extended over a range of $2.1 \mathrm{~km}$ at stack shot station $\mathrm{I}$ and $2.4 \mathrm{~km}$ at station 2 (Fig. 1). Because of limitations of recording capacity, 24 shots were recorded from station 1 and 36 from station 2. As a result, $9600-14400$ traces were recorded for each "stack shot" station. The experiment thus designed generated a significant amount of overlap of raypaths, which allows improvement of the $\mathrm{S} / \mathrm{N}$ ratio by appropriate trace stacking algorithms, discussed below.

\section{Trace stacking algorithms}

We define a receiver gather as the collection of all traces recorded at one receiver, a shot gather as the collection of all traces from one shot, and an offset gather as the collection of all traces falling within a specified range of shot-receiver distances. These definitions are compatible with the terminology commonly used in processing of near vertical-incidence reflection seismic data. Unlike the common-mid-point (CMP) concept used in reflection data processing, the stacking of CMP 

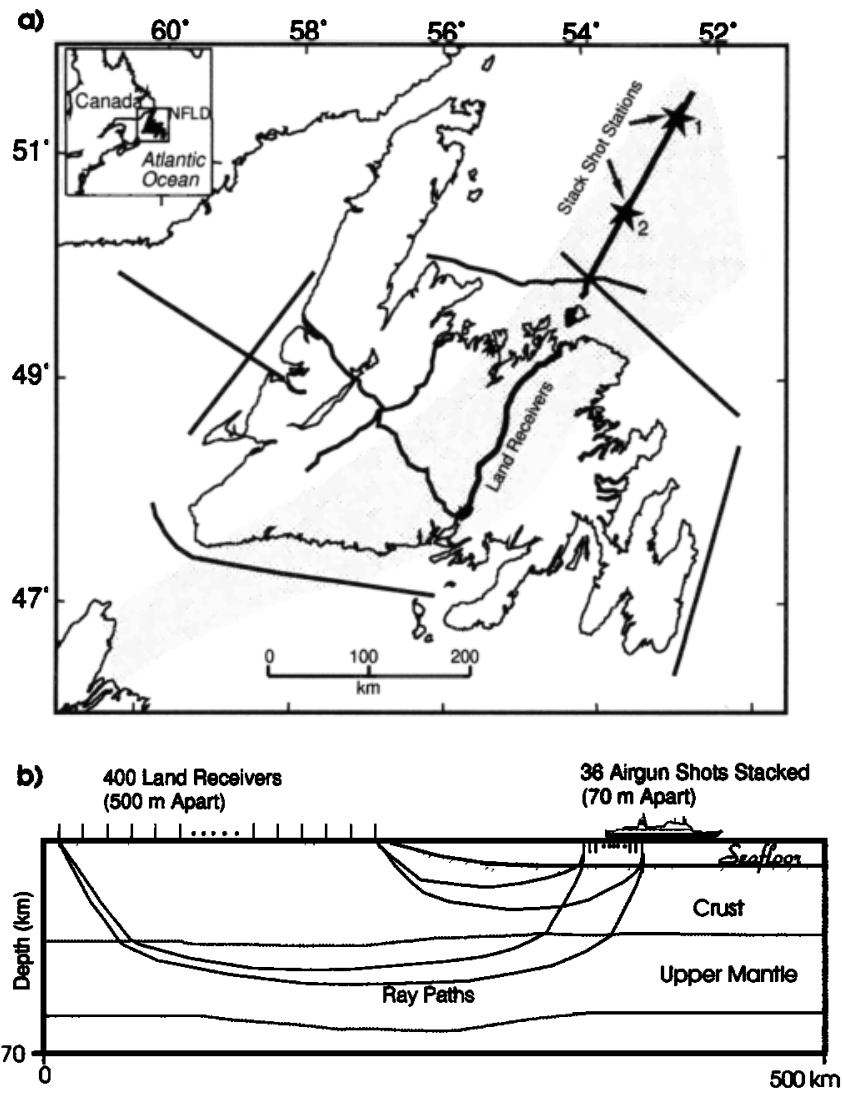

Figure 1. (a) Location map showing where the "stack shot" experiment was conducted. Shaded area delineates a banded zone of lower crustal reflectivity within the centre of the Appalachian orogenic belt (Marillier et al.. 1994). The thick solid north-south line crossing Newfoundland shows the locations of the land receivers, and the locations of the two stack shot stations are shown as stars. Thin solid lines are other refraction profiles. (b) Experiment setup and typical seismic raypaths. The airgun shots are aligned with the profile made by the seismic recorders on land.

gathers is not practically usable in this wide-angle experiment primarily because the geometry of seismic raypaths is heavily dependent on velocity variations at large offsets (say $>100 \mathrm{~km}$ ).

An obvious way of stacking the overlapped "stack shot" data is to sum all traces in each receiver gather along an optimal slope in the time-offset domain (say $8 \mathrm{~km} / \mathrm{s}$ ); we refer to this as the "simple" stack method (Fig. 2). This approach assumes that seismic arrivals in each receiver gather are aligned with little lateral variation. However, our data show that amplitudes, and possibly travel-times, may change irregularly even within a small aperture receiver gather. This variation may be due to lateral variation in the shallow structure below a "stack shot" site, and/or offset-dependent amplitude variations arising from the deep structure.

Another problem with the "simple" stack is the attenuation of signals with apparent velocities differing from the chosen linear moveout velocity $(8 \mathrm{~km} / \mathrm{s}$, typical mantle velocity). Stacking at $8 \mathrm{~km} / \mathrm{s}$ at a source array aperture of $2 \mathrm{~km}$, with a signal at a dominant frequency of $-8 \mathrm{~Hz}$, will pass signals with apparent velocities of $6.4-10.7 \mathrm{~km} / \mathrm{s}$ (maximum phase difference of half a period). This will include most of the P-wave crustal and mantle phases of significant amplitude at the ranges included here. However, stacking at this velocity will only be effective in a narrower range of 7.1-9.2 km/s (maximum phase difference
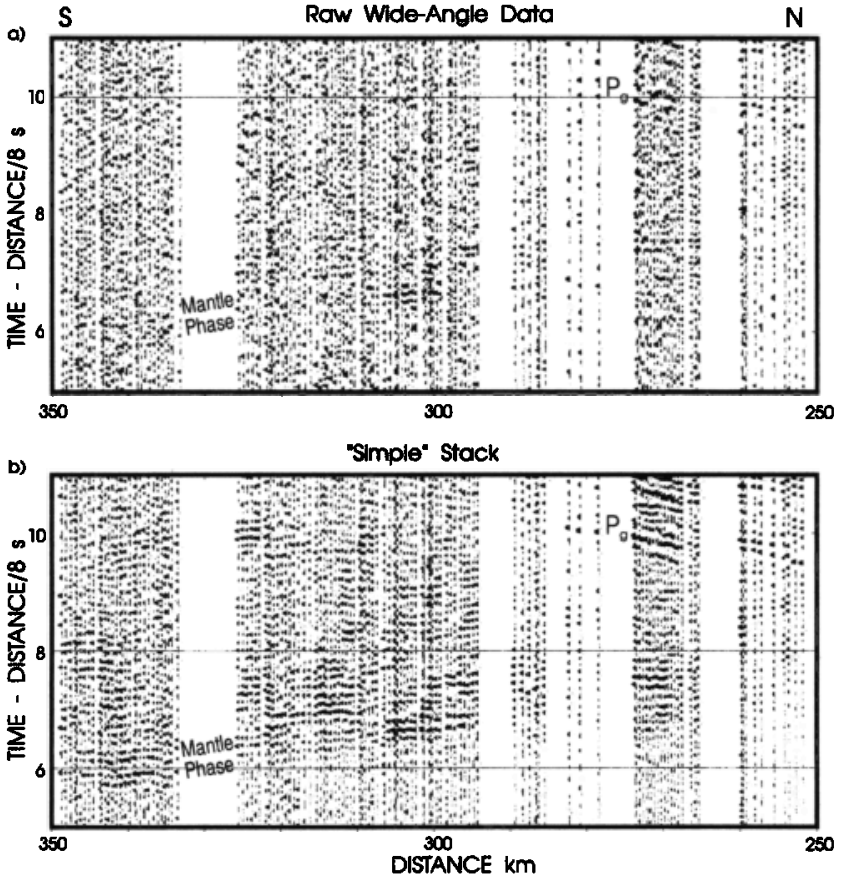

Figure 2. (a) Single shot gather for offsets between 250 and $350 \mathrm{~km}$. $\mathrm{Pg}$ is a refraction from the upper/mid crust. (b) "Simple" stack of 36 shot gathers at an aperture of $2.4 \mathrm{~km}$ along an $8 \mathrm{~km} / \mathrm{s}$ slope. For comparison purposes, all traces are normalized to a common maximum amplitude. The "Mantle Phase" is an arrival from within the upper mantle.

of a quarter period), so that stacking of this simple kind may not be ideal. A coherency stack generated by search for maximum coherency (Chian and Louden. 1992) over a wider range of apparent velocities would avoid this particular problem but (i) it would still be susceptible to inefficiencies generated by the previously mentioned irregularities in the receiver gathers; and (ii) it would be computationally much more intensive.

The smaller the offset range within a receiver gather, the less likely lateral variations are to adversely affect the stack. Instead of stacking all the traces within each receiver gather, we divide the traces into bins, each of $0.5 \mathrm{~km}$ offset aperture, and stack the traces within these bins along an $8 \mathrm{~km} / \mathrm{s}$ slope. The 2.1 (or 2.4)

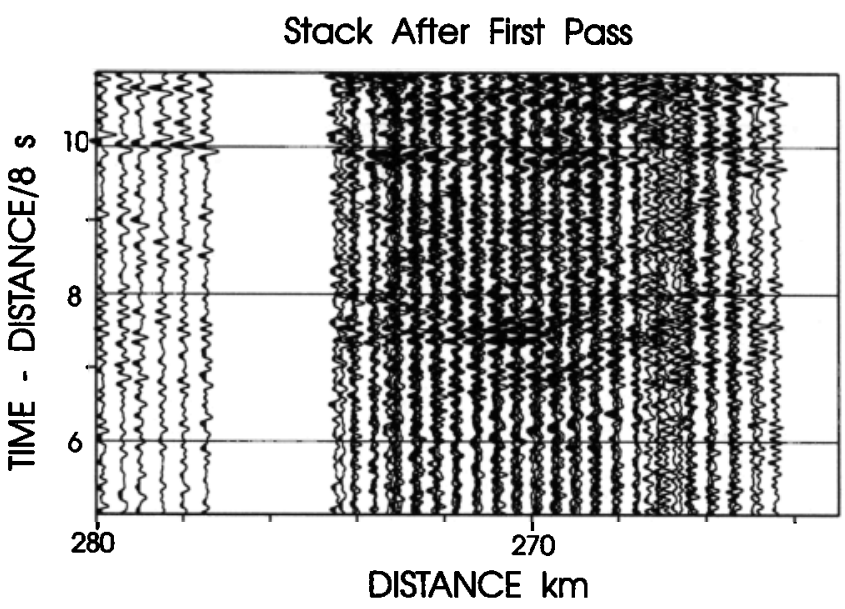

Figure 3. Intermediate output after the first-pass stack in the two-pass stacking technique. Each trace is the output of a 500 $\mathrm{m}$ aperture stack from the receiver gather domain. Traces from adjacent receivers overlap and group in clusters. 
$\mathrm{km}$ source aperture produces data in five bins, and each bin produces one stacked trace. In Figure 3, the five stacked traces from all receiver gathers are plotted as a function of sourcereceiver offset. Because the total offset range (2.1-2.4 km) of each receiver gather by far exceeds the receiver spacing $(\sim 500$ $\mathrm{m})$, traces from different receivers overlap each other. Because the stacking aperture is almost equal to the receiver spacing, clusters of traces build up, within which each trace is from a different receiver. A second-pass stack of all the traces within each cluster produces output traces spaced at $\sim 0.5 \mathrm{~km}$ (Fig. $4 b$ ). The apparent-velocity pass-band for stacking an $8 \mathrm{~Hz}$ signal at $8 \mathrm{~km} / \mathrm{s}$ over a $500 \mathrm{~m}$ aperture is $5.2-16 \mathrm{~km} / \mathrm{s}$; and the band of effective stacking is $4-8 \mathrm{~km} / \mathrm{s}$. The steps involved in the two-pass stack method can be summarized as follows.

1. Sort data into receiver gathers and organize the traces of each receiver gather according to increasing shot-receiver offsets, frequency filter at $3-10 \mathrm{~Hz}$.

2. Pre-processing: edit the traces, correct for timing errors within each receiver gather, adjust for small time delays between adjacent receivers, remove anomalous high-amplitude noise.

3. First-pass stack: stack traces with a $0.5 \mathrm{~km}$ offset aperture within each receiver gather.

4. Sort output traces from step 3 of all receiver gathers according to increasing shot-receiver offsets. This produces an offset gather, primarily composed by trace clusters at $\sim 0.5 \mathrm{~km}$ intervals. All traces in each cluster are from different receivers.

5. Second-pass stack: stack traces in each trace cluster. If no cluster evident, stack every $0.5 \mathrm{~km}$ (referred to as a stacking window). If a stacking window includes a data gap wider than $0.2 \mathrm{~km}$. the window is considered to include two clusters, and the window width is reduced to include only single clusters.

a)
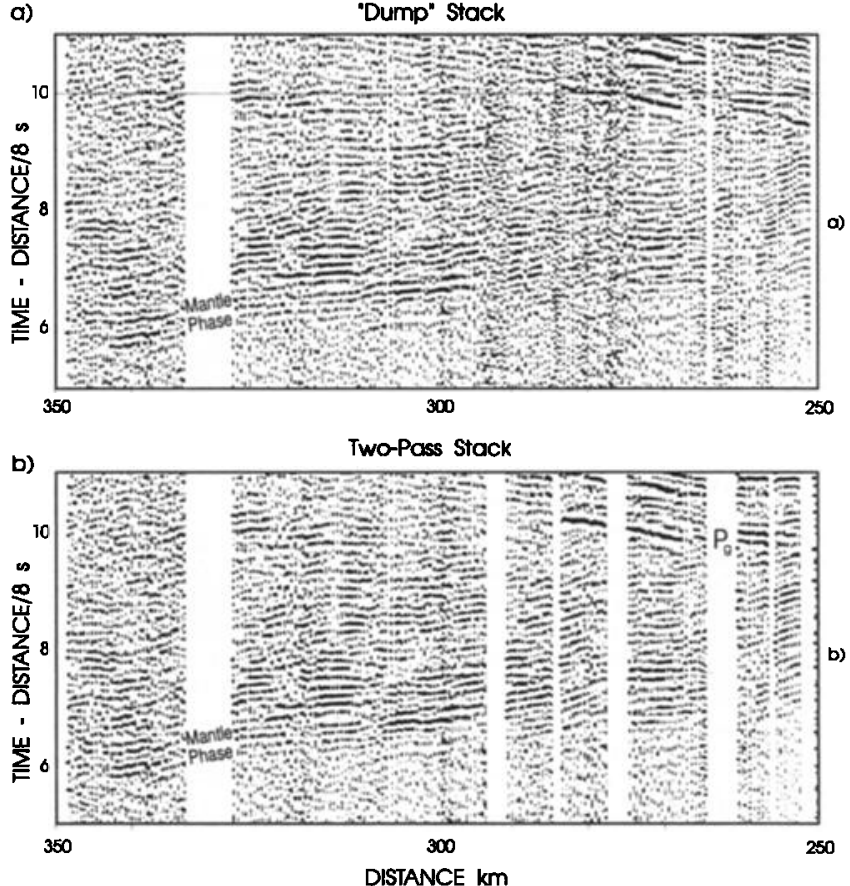

Figure 4. (a) "Dump" stack for all the traces in the 36 shot gathers, with no trace editing and no corrections applied. (b) Two-pass stack for the same data set, with trace editing and corrections applied. For comparison purposes, all traces are normalized to a common maximum amplitude. At places where receivers are sparsely distributed (e.g. $275-290 \mathrm{~km})$, this stack generates more traces than the "simple" stack (Fig. 2b).
During each step, a close user interaction with all the intermediate processing results is required to guarantee the best stacking results. In step 3, for example, traces with very high amplitude noise have to be removed to avoid masking of signals in the stack. Irregular time delays (in general small) due to instrumental problems of the receivers or statics effects were occasionally observed between two adjacent receiver gathers. For these gathers, time delays were computed by crosscorrelating the traces from one receiver gather with those from the next receiver gather at the same offsets, and the results used to correct the delayed traces.

The two operations of the two-pass stack can be merged into one, in which all traces are sorted according to shot-receiver offsets, without consideration of the receiver or shot to which they belong. These data would be sorted in $500 \mathrm{~m}$ "bins" and stacked. We call this method the "dump" stack (Fig. 4a). In theory, the "dump" stack is identical to the two-pass stack. However, in practice the latter is better. Output from the first-pass stack clearly shows whether a particular receiver is "bad", and such receivers can be corrected or eliminated. This is not possible in the dump stack. Furthermore, sorting of the receiver gather which is required by the first-pass stack and the sorting of the offset gather required by the second-pass stack allows viewing, identification, and correction of possible timing errors of seismic acquisition. Such trace editing helps enhance the signals as shown for example in Figure $4 \mathrm{~b}$ at $275-295 \mathrm{~km}$.

\section{Results}

To assess the effectiveness of the two-pass stack method the results can first be compared to the unstacked data. While recordings of one individual airgun shot at 254 to $256 \mathrm{~km}$ offsets show clear seismic arrivals, at larger offsets the $S / N$ ratio $(\leq 1)$ considerably deteriorates (Fig. 2a). In the "simple" stack (Fig. 2b), the signal has been significantly enhanced. The average $\mathrm{S} / \mathrm{N}$ ratio in this section can be visually estimated to be of the order of 3 . This corresponds to an increase in $\mathrm{S} / \mathrm{N}$ ratio which is slightly less than the theoretical value of 5-6 $\left(=n^{12}\right.$, with $\mathbf{n}$ the total number of traces in the stack lying in the range of 24-36).

A bigger improvement in $\mathrm{S} / \mathrm{N}$ ratio is observed in the two-pass stack (Fig. 4b). However, the advantage of this method is not just a matter of $S / N$. When signals of different apparent velocity cross, the "simple" stack tends to merge the phases while the two-pass stack retains them as separate events. The adoption of a smaller aperture is thus similar to applying a less restrictive velocity filter and tends to retain information with better fidelity. The overall result is that seismic events that are barely discernible on the single shot gather (Fig. 2a) can now be followed over many tens of kilometres in the two-pass stack section (Fig. 4b). The two-pass stack technique also produces a more continuous section with smaller gaps between output traces because the first phase stacking is done in 5 bins with different offsets. The result is to make it easier to correlate events across the sections (compare Fig. $2 \mathrm{~b}$ and Fig. $4 \mathrm{~b}$ at 277 $292 \mathrm{~km}$ ). It should be noted that after the two-pass stack, there is no redundancy in the data. The display quality can be improved further by additional trace stacking algorithms such as coherency mixing (Chian and Louden. 1992). Figure 5 shows the two profiles from the "stack shot" stations northeast of Newfoundland after being thus processed. It is evident that the technique generates high-quality seismic sections, while extending the offsets of usable data from conventional $200 \mathrm{~km}$ to over $450 \mathrm{~km}$. 

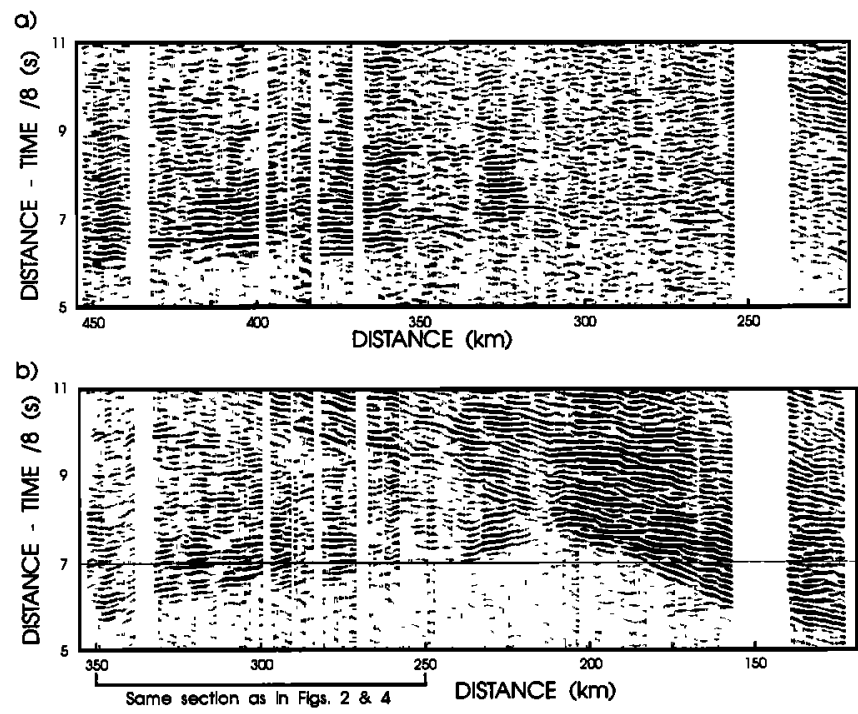

Figure 5. Coherency mixing of the two-pass stacked profiles for stations 1 (a) and 2 (b). Mixing is performed along the direction of maximum coherency measured across 5 traces within a time window of $0.15 \mathrm{sec}$.

\section{Discussion}

The two-pass stacking procedure can fail to enhance the data if there are major structural complexities below the shot area and below the receivers, such that offset gathers show a scatter of arrival times for a given phase at one offset. Forcing such data through a "dump" stack would also produce an inefficient stack if the scatter were a significant fraction of the dominant period of the signal. This is not a problem in our case: if it were, then the two-pass stack adjusted if necessary for stacking at different velocities in each pass, would result in a stack yet more effective than either the "simple" or "dump" stack.

The geometry of our stack shot experiment can be generalized by the following relations: shot spacing $\sim 70 \mathrm{~m}$, receiver spacing $\sim 500 \mathrm{~m}$, and shot array aperture $\sim 2 \mathrm{~km}$. During processing of such data, a stacking aperture that is close to the receiver spacing should be used consistently for each phase of the two-pass stack. We have demonstrated that the results from such a design can be processed by our two-pass stack technique to successfully enhance wide-angle seismic signals. A comparison of Figures $2 \mathrm{a}$ and $4 \mathrm{~b}$ suggests that the 36 shot sequence improves the $\mathrm{S} / \mathrm{N}$ ratio by a factor of 6 . Phases barely discernible in the single airgun shot gather (Fig. 2a) become quite clear and correlatable over many tens of kilometres in the two-pass stack (Fig. 5).

\section{Conclusions}

By stacking shot arrays of about $2 \mathrm{~km}$ in length, we have demonstrated that wide-angle seismic surveys of continental crustal structure using marine airgun sources can be extended to provide good arrivals to offsets of over $450 \mathrm{~km}$. Previous experiments with airgun sources of up to 200 litres had usually been limited to observations of crustal phases, wide-angle reflections from the Moho and, occasionally, head waves $(\mathrm{Pn})$ from the top part of the mantle. Now, using the stack shot technique, the offsets of surveys can be more than doubled, and deeper mantle phases can be recorded.

Given the current reluctance, for environmental reasons, to use large single dynamite sources at sea, the stack shot technique offers opportunities to simulate such sources using smaller, environmentally "friendlier" sources. The approach could be slightly modified for stacking the energy from any small source, terrestrial or marine.

Acknowledgments. We would like to thank all the people who participated in the field experiment. Special thanks are due to the personnel on board the CSS Hudson who made the special shooting procedure possible. We thank lan Reid for the use of his seismic processing and display computer programs. We acknowledge the help of Brian Roberts (Geological Survey of Canada) in putting the data set together. and Jim Luetgert (US Geological Survey) who sent us copies of original field tapes and helped us read them. The reviews of K. Coflin, C. Hurich, B. Zelt and two anonymous referees are gratefully acknowledged. This work was supported by the Natural Sciences and Engineering Research Council of Canada (NSERC) and the Geological Survey of Canada (GSC), through their partnership in the Lithoprobe project and through research grants from NSERC (to J.H. and G.M. Quinlan). This paper is Lithoprobe Contribution 769 and GSC Contribution 41195.

\section{References}

Chian. D., and K.E. Louden. The structure of Archean/Ketilidian crust along the continental shelf of southwestern Greenland from a seismic refraction profile. Can. J. Earth Scl.. 29.301-313. 1992.

Hughes, S.. J. Hall. and J.H. Luetgert. The seismic velocity structure of the Newfoundland Appalachian orogen, J. Geophys. Res., 99, 13633-13653. 1994.

Marillier. F.. I Hall, S. Hughes, K. Louden, I. Reid, B. Roberts, R. Clowes. T. Coté, J. Fowler, S. Guest. H. Lu, J. Luetgert, G. Quinlan, C. Spencer, and J. Wright, Lithoprobe East onshore-offshore seismic refraction survey -constraints on interpretation of reflection data in the Newfoundland Appalachians. Tectonophysics. 232. 43-58. 1994.

Neprochnov. Yu.P.. V.V. Sedov, A.A. Ostrovskiy, and S.Y. Sokolov, Experimental findings on the accumulation of airgun seismic signals at the west coast of the Caspian Sea. Oceanology, 25(2), 245-248, 1985.

D. Chian. Department of Oceanography, Dalhousie University, Halifax NS, Canada B3H 3.55 (e-mail: qian 1 (a)ac.dal.ca)

J. Hall, Department of Earth Sciences. Memorial University of Newfoundland. St. Joln's NF. Canada AIB 3X5 (e-mail: jeremy(a)kean.ucs.mun.ca)

F. Marillier. Institute of Geophysics, University of Lausanne. College Propedeutique. 1015 Lausanne, Switzerland (email: fmarilli@ig.unil.ch)

(Received: March 4. 1996: accepted April 17. 1996) 